\title{
REFERENCES
}

Baird, D. (1945). F. Obstet. Gynaec. Brit. Emp. 52, 339.

Barcroft, J. (1946). Researches on Pre-natal Life. Oxford: Blackwell Scientific Publications.

Beilly, J. S. \& Kurland, I. I. (1945). Amer. F. Obstet. Gynec. 50, 202.

Burke, B. S., Harding, V. V. \& Stuart, H. C. (r 943). F. Pediat. 23, 506.

Clatworthy, H. W. Jr. \& Anderson, R. G. (1944). Amer. Y. Dis. Child. 67, 167.

Corner, G. W. (1944). Ourselves Unborn. London: Humphrey Milford.

Flexner, L. B., Cowie, D. B., Hellman, L. M., Wilde, W. S. \& Vosburgh, G. J. (1948). Amer. F. Obstet. Gynec. 55, 469 .

Hale, F. (1935). Amer. F. Ophthal. 18, 1087.

Hosemann, H. (1948). Arch. Gynäk. 176, 109.

Hecker, C. (1866). Quoted by J. Needham (1931) in Chemical Embryology, Vol. 3. Cambridge: University Press.

Lawrence, R. D. \& Oakley, W. (1942). Quart. F. Med. I1, 45.

Leitch, I. (r950). In Combined Text Book of Obstetrics and Gynaecology, ch. 7 [D. Baird, editor]. Fdinburgh: Livingstone.

Martin, W. J. (1930-I). Ann. Eugen., Camb., 4, 327.

Needham, J. (1942). Biochemistry and Morphogenesis. Cambridge: University Press.

Sontag, L. W. \& Wines, J. ( 1947). Amer. F. Obstet. Gynec. 54, 994.

Thomson, A. M. \& Thomson, W. (1948-9). Brit. F. Nutrit. 2, 290.

Walton, A. \& Hammond, J. (1938). Proc. roy. Soc. B, 125, 311.

Wallace, L. R. (1946). F. Physiol. ro4, 33 P.

Wallace, L. R. (1948). F. agric. Sci. 38, 93.

Warkany, J. (1945). Vitamins and Hormones, 3, 73.

\section{Secular Changes in Growth}

BY J. B. DE V. WEIR, Institute of Physiology, University of Glasgow

This contribution is printed by title only since it has been submitted for publication as an original paper. 